ORIGINAL ARTICLE

\title{
Diagnostic Accuracy of a New Sonographic Classification System in Diagnosing Malignant Thyroid Nodules
}

MUHAMMAD AMIN ${ }^{1}$, MAHWISH ZAHRA ${ }^{2}$, MUSTAFA ALI SIDDIQUI ${ }^{3}$, RIFFAT KAMAL ${ }^{4}$, SAHAR JAVED 5 , AMENA

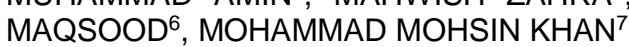

${ }^{1}$ Assistant Professor Radiology, Children Hospital and Institute of Child Health Multan

${ }^{2}$ Senior Registrar Radiology Children Hospital and Institute of Child Health Multan

${ }^{3}$ Professor Radiology CPEIC Multan

${ }^{4}$ Senior Registrar CPEIC Multan

${ }^{5}$ Senior Registrar Paediatric Radiology Children Hospital and Institute of Child Health Multan

${ }^{6}$ Medical Officer Radiology Children Hospital and Institute of Child Health Multan

${ }^{7}$ Professor Community Medicine NM\&DC

Corresponding author: Dr. Muhammad Amin, Cell: 0333 6121755,Email: i@amin.ai

\begin{abstract}
Objective: To evaluate the validity of new ultrasound classification in diagnosing malignant thyroid nodule keeping fine needle aspiration cytology (FNAC) as gold standard.

Materials and Methods: This validation study was carried out at hospital from September 06-2020 to March2021 in Children Hospital and Institute of Child Health Multan .A total number of 114 patients who presented with inflamed thyroid nodules were included. General information regarding patient like name, age and gender was documented for data collection. Ultrasonography was done using Linear 7.5 MHz and curvilear $3.5 \mathrm{MHz}$ transducer all patient in supine position and comfortable setting. Features of ultrasound were recorded. Patients having U4-U5 score were labelled as having malignant lesions. All cases were followed up by fine needle aspiration and corresponding histopathology report.

Results: Mean age of patients was $46.06 \pm 10.34$ years. Out of 114 patients, $83(72.81 \%)$ patients were female and only 31 (27.19\%) were male. On ultrasonography, malignancy was diagnosed in only $24(21.05 \%)$ patients, and remaining 90 (78.95\%) were diagnosed of having benign lesions. On histopathology, malignancy was diagnosed in 25 (21.93\%) lesions, while remaining $89(78.07 \%)$ patients were diagnosed of having benign lesions. Sensitivity of ultrasound U-score in diagnosing malignant thyroid nodules was $88 \%$, specificity was 97.8\%, PPV was $91.7 \%$ and NPV was $96.7 \%$.

Conclusion: New sonographic classification system has a reliable accuracy for differentiating malignant from benign solid thyroid nodules. So it can be used as preferred radiological scoring system for initial investigation for solid thyroid nodules.

Keywords: Thyroid nodules, ultrasound U-Score, Histopathology, Malignancy, Diagnostic accuracy.
\end{abstract}

\section{INTRODUCTION}

Thyroid nodules are a common finding in clinical practice. ${ }^{1}$ Depending on size, it can be seen as neck swelling and palpated by clinical examination in about 3 to $7 \%$ of general population. $^{2}$ Diagnosis by ultrasound neck or as an incidental finding during carotid doppler increases the incidence from 13 to $67 \%$ that further rises in older age..$^{1,3}$

Thyroid nodules are found accidently in 3 to $7 \%$ adult population on clinical examination, among these $30-70 \%$ have clinically detectable thyroid nodules on ultrasonographic (US) examination. ${ }^{4}$ Among these only $10 \%$ of the nodules are diagnosed as malignant. ${ }^{5}$

In Pakistan, thyroid diseases are common probably due to iodine deficiency. According to UNICEF report, nearly about $70 \%$ of the Pakistani population is at risk of developing thyroid diseases due to iodine deficiency. ${ }^{1}$ The prevalence of thyroid cancer in Pakistan is about $1.2 \%$ among all patients of thyroid nodules. ${ }^{1}$

Fine needle aspiration cytology (FNAC) has been the counter stone for discriminating malignant from benign thyroid nodules and therefore reduce the need for unwanted surgical procedures. In 2014, BTU guidelines suggested to use ultrasound based $U$ classification system for differentiation of thyroid nodules. ${ }^{6}$

Although a number of investigative and diagnostic modalities like computed tomography, magnetic resonance imaging and radio-nucleotide thyroid scan are available, ultrasound is main modality used for not only initial assessment but also for characterization of thyroid nodule upon which number of classification systems are based. ${ }^{7}$ It has an edge over others as it is cost effective, radiation free, available and dynamic. ${ }^{7}$ In the study, the accuracy of a new thyroid nodule classification system was assessed that can facilitate in directing the need for fine needle aspiration.

\section{MATERIALS AND METHODS}

This validation study was carried out in Children Hospital and Institute of Child Health Multan from September 062020 to March-2021. A total number of 114 patients who presented with inflamed thyroid nodules of age 19 to 60 were included. Patients with cystic nodules, and postoperative thyroid lesions were excluded. General information regarding patient like name, age and gender was documented for data collection. Informed consent was taken from the patients.

Sample size for this study is calculated by taking estimated prevalence of malignant lesions $20.7 \%{ }^{1}$ among patients of inflamed thyroid nodules. And by taking expected sensitivity of ultrasonography $90.3 \%,{ }^{1}$ specificity of $94.1 \%^{1}$ in diagnosing malignant lesions, ${ }^{1}$ by taking 
desired precision level of $10 \%$ for sensitivity and $6.0 \%$ for specificity.

Ultrasonography was done using Linear $7.5 \mathrm{MHz}$ and curvilear $3.5 \mathrm{MHz}$ transducer all patient in supine position and comfortable setting. Features of ultrasound were recorded. Patients having U4-U5 score were labelled as having malignant lesions. the $U$ score was calculated according to the BTA guidelines.

After US, biopsy specimens were taken under ultrasonography guidance. Specimens were sent for cytology reporting. In samples with suspicion of malignancy. Histopathology was further done to diagnose malignant tumors.

Data was entered and analyzed using SPSS version 20.0. Mean and standard deviation was used for quantitative variable like age. Qualitative variables like gender, presence of malignancy on ultrasonography (U4-U5) score, and presence of malignancy on histopathology reporting. $2 \times 2$ contingency table was used to calculate sensitivity, specificity, positive predictive value, and negative predictive value of ultrasonography by taking histopathology reporting as gold standard.

\section{RESULTS}

Mean age of patients included in this study was $46.06 \pm 10.34$ years. Out of 114 patients, 83 (72.81\%) patients were female and only $31(27.19 \%)$ were male.

On ultrasonography, malignancy was diagnosed in only $24(21.05 \%)$ patients, and remaining 90 (78.95\%) were diagnosed of having benign lesions. On histopathology, malignancy was diagnosed in 25 (21.93\%) lesions, while remaining $89(78.07 \%)$ patients were diagnosed of having benign lesions.

Sensitivity of ultrasound U-score in diagnosing malignant thyroid nodules was $88 \%$, specificity was $97.8 \%$, PPV was $91.7 \%$ and NPV was $96.7 \%$ (Table 1 ).

Table 1. Diagnostic Accuracy of Ultra-Sonography $U$ Score in Diagnosis of Malignant Thyroid Nodules Taking Histopathology as Gold Standard.

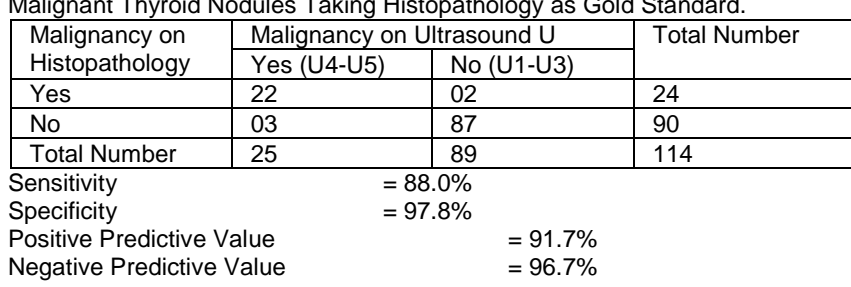

\section{DISCUSSION}

Thyroid nodules are quite common particularly in females and ultrasound is often the first radiological examination asked. Ultrasound was believed to be useful just in separating solid from cystic nodules, but higher resolution probes and doppler scanning has carried ultrasound to a higher level by denoting certain factors which have become better appreciable by the new machinery. ${ }^{8}$

Because no single sonographic feature is dependable in separating benign from malignant thyroid nodules and an overlap exists between them. So British thyroid association, in 2014, came up with a $U$ classification system which compiled all the research done on thyroid nodules and classified the nodules based on their knowledge of features associated with proven cases of benign and malignant nodules into five categories U1 being normal and U5 containing features which were most consistently associated with malignancy. This tool will help the radiologist to further guide for the need of FNAC on selected patients as it is not possible to target all the nodules to aspiration which can be both a financial and physical burden. ${ }^{9}$ So, nodules classified into U2 were always benign so they just needed to be reported as such, no intervention was needed. But categories U4 and U5 had features which either in solitude or multitude were associated with malignancy, so had to be aspirated. U3 however was the class which although was the commonest yet hardest to predict, as the risk of malignancy in this class was low but nonetheless not ignorable. ${ }^{10,11}$

So we carried out this study in our department to assess the diagnostic accuracy of this classification system in our set up and thus benefit from the study reducing both the patient anxiety and morbidity. We have classified the patients on the basis of $U$ classification into two groups. Group one consisted of $\mathrm{U}-1$ nodules to $\mathrm{U}-3$ nodules defined as benign cases and U4 and U5 classified nodules were considered as malignant cases.

In our study, the sensitivity of ultrasound U-score in diagnosing malignant thyroid nodules was $88 \%$, specificity was $97.8 \%$, PPV was $91.7 \%$ and NPV was $96.7 \%$.

A study conducted by Ghani et al. concluded that ultrasound $U$ score is $100 \%$ sensitive, $91.3 \%$ specific, has PPV of $60 \%$ and NPV $100 \%$ for diagnosis of malignant lesions. This study aims to evaluate the reliability and validity of the new classification of thyroid in predicting thyroid malignancy using sensitivity and specificity of ultrasound taking findings of histopathology as the reference standard. ${ }^{12}$

Sanaullah et al. reported that ultrasound $U$ score is $90.3 \%$ sensitive and $94.1 \%$ specific for diagnosis of malignant tumors, with prevalence of malignant nodules $20.7 \%$ and that of benign nodules $79.3 \%$. $^{1}$

Ahmad et al. also reported similar results, they included 210 patients of thyroid nodules to determine the diagnostic accuracy of ultrasound U-score, they reported that U-score is $90.24 \%$ sensitive, $94.67 \%$ specific, and had PPV of $80.43 \%$ and NPV $97.56 \% .^{13}$

A study conducted by Akhter et al. on diagnostic accuracy of ultrasound U-score reported sensitivity of $80 \%$, specificity $34 \%$, positive predictive value $100 \%$ and negative predictive value $90 \% .^{14}$

Taking everything into account, Ultrasound $U$ classification system has a high indicative precision in distinguishing and segregating thyroid nodules on premise of features like echogenicity, edges, small scale calcifications shape and vascularity. Radiologists' must be acquainted with these signs on ultrasound that guide to separate benign from malignant lesions and for right application of FNAC. So that, diagnosis can be made more cost effective even for the low socio economic population and avoid unnecessary aspirations but expedite them when needed.

\section{CONCLUSION}

Diagnostic accuracy of a new sonographic classification system in the differentiation of benign from malignant solid thyroid nodules, while keeping FNAB as gold standard was 
very high and is recommended for diagnosis of malignant solid thyroid nodules.

\section{REFERENCES}

1. Sanaullah, Anwar J, Soomro JA. Accuracy of a new sonographic classification system in differentiating benign from malignant solid thyroid nodules. Pak Armed Forces Med J. 2017;67(3):401-06.

2. Hatem M, Farheen S. Thyroid nodule size as an indicator for surgery. Int Surg J. 2018;5(7):2401.

3. Alam T, Khattak Y, Beg M, Raouf A, Azeemuddin M, Khan A. Diagnostic accuracy of ultrasonography in differentiating benign and malignant thyroid nodules using fine needle aspiration cytology as the reference standard. Asian Pacific J Cancer Prev. 2014;15(22):10039-43.

4. Frates MC, Benson CB, Charboneau JW, Cibas ES, Clark $\mathrm{OH}$, Coleman BG, et al. Management of thyroid nodules detected at US: Society of Radiologists in Ultrasound consensus conference statement. Radiology. 2005;237(3):794-800.

5. Middleton WD, Teefey SA, Reading CC, Langer JE, Beland MD, Szabunio MM, et al. Multi-institutional analysis of thyroid nodule risk stratification using the American College of Radiology Thyroid Imaging Reporting and Data System. Am J Roentgenol. 2017;208(6):1331-41.

6. Perros P, Boelaert K, Colley S, Evans C, Evans RM, Gerrard $\mathrm{Ba} \mathrm{G}$, et al. Guidelines for the management of thyroid cancer. Clin Endocrinol (Oxf). 2014;81(suppl. 1):1-122.

7. Zevallos JP, Hartman CM, Kramer JR, Sturgis EM, Chiao EY. Increased thyroid cancer incidence corresponds to increased use of thyroid ultrasound and fine-needle aspiration: a study of the Veterans Affairs health care system. Cancer. 2015;121(5):741-6.

8. Leenhardt L, Tranquart F, Tramalloni J. Advances in thyroid ultrasound for detection and follow-up of thyroid malignancies. Thyroid cancer: from emergent biotechnologies to clinical practice guidelines. 2016;19:99106.

9. Wettasinghe MC, Rosairo S, Ratnatunga N Wickramasinghe ND. Diagnostic accuracy of ultrasound characteristics in the identification of malignant thyroid nodules. BMC Res Notes. 2019;12(1):193.

10. Kim DW, Lee EJ. Differentiation of Benign and Malignant Solid Thyroid Nodules Using an Ultrasonography Classification System. Ultrasound Med Biol. 2011;37(8):S130.

11. Al-Ghanimi IA, Al-Sharydah AM, Al-Mulhim S, Faisal S, AlAbdulwahab A, Al-Aftan M, Abuhaimed A. Diagnostic accuracy of ultrasonography in classifying thyroid nodules compared with fine-needle aspiration. Saudi J Med Med Sci. 2020;8(1):25-9.

12. Suhaimi SN, Sridharan R. Reliability of the ultrasound classification system of thyroid nodules in predicting malignancy. Med J Malaysia. 2018;73(1):9-15.

13. Ahmad MI, Qasmi IM, Dildar N, Nadeem SF, Nadeem K. Validity of a new ultrasound (USG) classification system for differentiating between benign and malignant solid thyroid nodules. Pak J Radiol. 2016;24(2):51-5.

14. Akhter T, Shahid K, Afzal U. Diagnostic Accuracy of Ultrasound U Classification System of Thyroid Ultrasound in Predicting Thyroid Malignancy by Using Histopathology as Gold Standard. Ann PIMS-Shaheed Zulfiqar Ali Bhutto Med Uni. 2018;14(3):222-6. 